\title{
Experimental study of energy exchanges between two coupled granular gases
}

\author{
J.-Y. Chastaing, J.-C. Géminard, and A. Naert \\ Université de Lyon, Ens de Lyon, Université Claude Bernard, CNRS, Laboratoire de Physique, F-69342 Lyon, France
}

(Received 10 September 2016; published 7 December 2016)

\begin{abstract}
We report on the energy exchanges between two granular gases of different densities coupled electromechanically by immersed blades attached to dc motors. Zeroing the energy flux between the two subsystems, we demonstrate that an immersed blade is a convenient way to assess the properties of the granular gases, provided that the dissipation in the motor is properly taken into account. In addition, when the two gases have different densities, the fluctuations of the energy flux are asymmetric, very intermittent, and with most probable zero flux. We show that, for weak coupling, the main features of the energy exchanges can be explained considering the fluctuations of the two subsystems.
\end{abstract}

DOI: 10.1103/PhysRevE.94.062110

\section{INTRODUCTION AND BACKGROUND}

During the last decades dissipative systems have been widely studied and compared to equilibrium states. One can think about either systems slowly relaxing toward equilibrium or maintained in nonequilibrium steady state (NESS) by external forces [1,2]. A NESS is achieved when the energy losses, due to an intrinsic dissipative mechanism, are balanced by energy injection. Typical examples of NESS are, among others, hydrodynamic turbulence [3,4], turbulent thermal convection [5], electronic circuits [6-8], wave turbulence $[9,10]$, and molecular machines [11-14]. In granular materials, a NESS can be achieved by vibrating a collection of solid particles in a container. A granular gas is obtained when energy dissipated via inelastic collisions (between the beads or with the walls) is compensated by that injected to the particles by vibration [2,15-19].

In the present paper, we address experimentally the question of the energy flux between two such systems. Two independent granular gases of different densities are produced by vibrating vessels containing beads. A blade allowed to rotate around its vertical axis is immersed in each of the systems. Their dynamics is followed thanks to dc motors. Connecting the motors one to another makes convenient the coupling between the two NESS.

Lecomte et al. showed experimentally that the average energy flux is proportional to the difference between the variances of the blades' angular-velocity distributions [20]. Here, we focus on the statistical properties of the systems, and on the instantaneous energy exchanges between them, at zero average energy flux in the stationary state.

\section{EXPERIMENTAL SETUP}

The experimental device (Fig. 1) has been previously used to study the energy flux between two granular gases at different temperatures [20]. The core principle of the experiment consists in coupling two granular gases of different densities. The first gas is considered as the reference gas: its temperature is kept unchanged. The second one is of variable density. In order to study the influence of the gas density at constant granular temperature, we impose the mean energy flux between the two gases to be zero.
Each part of the device is made of an aluminum vessel, vibrated vertically thanks to a permanent-magnet shaker (Bruel \& Kjaer, model 4809) driven by the sinusoidal current (frequency $f=40 \mathrm{~Hz}$ ) from a power amplifier (Kepco, model BOP50-4M) fed by a low-frequency generator (Agilent, model $3352 \mathrm{X}$ ). The internal volume of the vessel is mainly cylindrical, $5 \mathrm{~cm}$ in diameter and $6 \mathrm{~cm}$ in height, but note that the bottom is machined slightly conical (typical angle, $10 \mathrm{deg}$ ) in order to enhance the transfer of momentum from vertical to horizontal when the beads collide with the latter. The reference gas is composed of $N_{1}=374$ stainless-steel beads, $3 \mathrm{~mm}$ in diameter (typical weight, $0.1 \mathrm{~g}$ ), the second of $N_{2}$ (from 20 to 374) beads.

In each gas, a blade $(2 \mathrm{~cm} \times 2 \mathrm{~cm}$, thickness $0.25 \mathrm{~mm}$, iron $)$ is placed vertically, centered, the lower edge a few millimeters above the bottom. Note that the height of the blades is the same in both systems, kept constant all along the present study. To do so, at the top the blade is wedged at its center on the axis of a dc micromotor (Maxon, RE 10118 386, nominal power $0.75 \mathrm{~W}$, voltage $6 \mathrm{~V}$, and internal resistance $r \simeq 21.2 \Omega$ ) attached to the upper cover of the vessel. The rotor is ironless, which minimizes the inertia, and precious metal brushes improve the electrical contact with the commutator. In our experimental configuration, the terminals of each motor are connected to each other by means of a resistor $R=1 \mathrm{k} \Omega$ (Fig. 2). The dc motors can be used reversibly as motor or generator. As a motor, the torque, $\Gamma_{i}=\alpha I$, is proportional to the current $I$, the factor $\alpha$ representing all the physical characteristics of the motor, like the number of poles, coils, and turns, magnetic field of the permanent magnets, and geometric factors. As a generator, a voltage, $e_{i}=\alpha \dot{\theta}_{i}$, proportional to the angular velocity $\dot{\theta}_{i}$ is induced. Notice here that the coefficient $\alpha=4.27 \times 10^{-3} \mathrm{~V} \mathrm{~s} / \mathrm{rad}$ is unique. When momentum is transferred to one blade by the surrounding beads in one reservoir, a voltage is applied to the circuit. This voltage induces a current in the circuit and, thus, a torque is applied to the other blade. Finally, note that the electromechanical devices being reversible, the process occurs in both directions with the same characteristics. The coupling between the two blades can be tuned by changing the value of $R$, which controls the voltage-to-current ratio. We chose a large value of $R$, such that the coupling remains weak. We checked experimentally, by opening the circuit, that the coupling does not significantly alter the distribution of the angular velocities $\dot{\theta}_{i}$. 


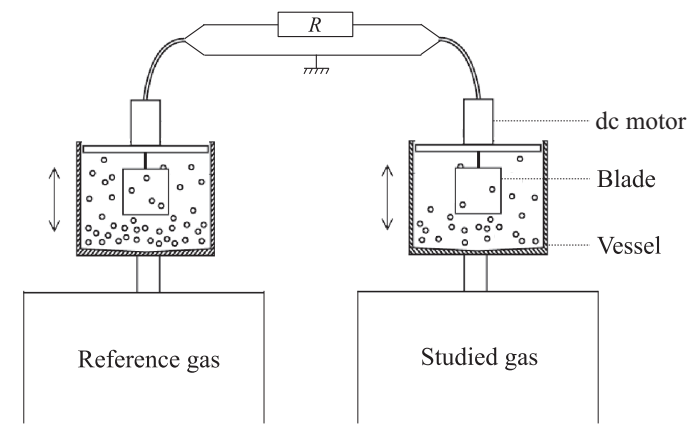

FIG. 1. Sketch of the experimental setup.

On can define the average kinetic energy of the blade, thermalized by the granular heat bath:

$$
E_{k, i} \equiv \frac{1}{2} M\left\langle\dot{\theta}_{i}^{2}\right\rangle,
$$

where $M$ stands for the total moment of inertia of the parts in solid rotation (blade + rotor of the motor, $M=3.33 \times$ $10^{-8} \mathrm{~kg} \mathrm{~m}^{2}$ ). The quantity $E_{k, i}$ provides an energy scale for the blade immersed in gas $i$. In what follows, after having checked that the coupling does not alter the measurements, we report values of $E_{k, i}$ measured on the coupled subsystems. The question we try to answer is to know to what extent $E_{k, i}$ accounts for the temperature of the granular gas $i$.

The terminals of each of the motors are connected to an analog-to-digital converter (National Instruments, NI PXI 4462) in order to measure voltages $u_{i}$. We have

$$
\begin{gathered}
I=\left(u_{1}-u_{2}\right) / R, \\
e_{1}=u_{1}\left(1+\frac{r_{1}}{R}\right)-u_{2} \frac{r_{1}}{R}, \\
e_{2}=u_{2}\left(1+\frac{r_{2}}{R}\right)-u_{1} \frac{r_{2}}{R},
\end{gathered}
$$

and define the energy flux

$$
\begin{aligned}
\Phi & \equiv \dot{\theta}_{1} \Gamma-\dot{\theta}_{2} \Gamma=I e_{1}-I e_{2} \\
& =\frac{1}{R+r_{1}+r_{2}}\left(e_{1}^{2}-e_{2}^{2}\right) .
\end{aligned}
$$

The experiment consists in studying the influence of the number of beads $N_{2}$ on the statistics of the blade velocity $\dot{\theta}_{2}$ (or equivalently $e_{2}$ ) and energy flux $\Phi$ at constant blade temperature $E_{k, 2}$. One reference (gas 1 ) is kept unchanged,

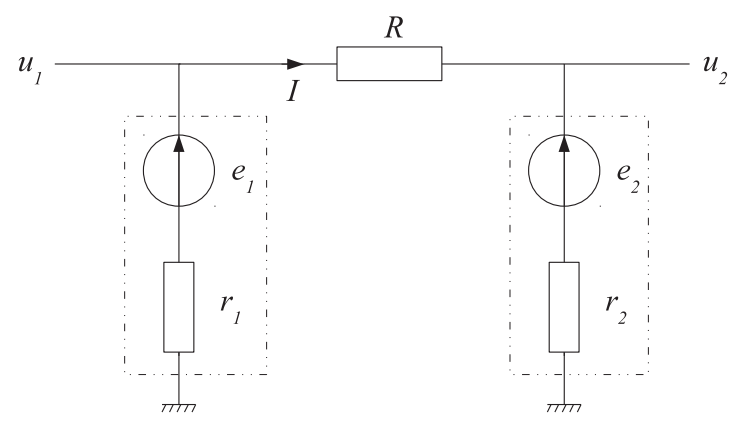

FIG. 2. Sketch of the electrical circuit.

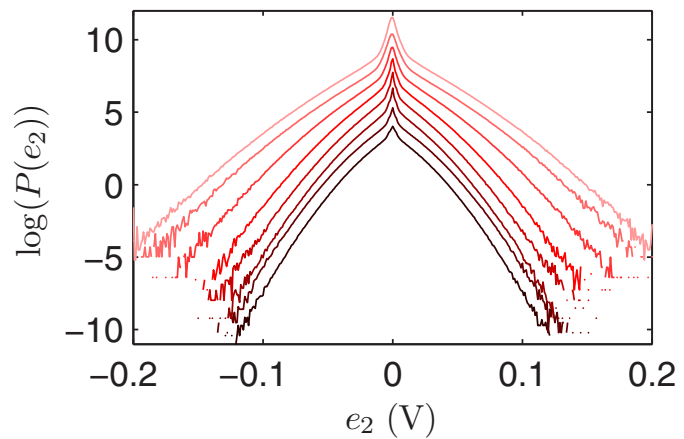

FIG. 3. Probability distribution functions $P\left(e_{2}\right)$ for several values of $N_{2}$ (from dark to light red: $N_{2}=374,249,167,111,74,50,30$, and 20 beads). Curves are shifted for clarity.

with $E_{k, 1}=4.5 \times 10^{-7} \mathrm{~J}$. Then, for various numbers of beads $N_{2}$, we impose the same temperature of the second blade by tuning the amplitude of the vibration so as to achieve a zeromean flux, $\langle\Phi\rangle=0$. In practice, we achieve $\langle\Phi\rangle \leqslant 0.02 \Phi_{\mathrm{RMS}}$, where $\Phi_{\mathrm{RMS}}$ is the root mean square of $\Phi$. Then, the system is shaken for several (up to 20) hours to ensure that the gas is in a steady state, and the voltages $u_{1}$ and $u_{2}$ are subsequently monitored for 20 hours at rate $f_{\mathrm{e}}=1024 \mathrm{~Hz}$. In the following, we report the results obtained with $N_{1}=374$ and $N_{2}=374$, $249,167,111,74,50,30$, and 20 beads.

\section{EXPERIMENTAL RESULTS}

\section{A. Blade velocity fluctuations}

\section{Experimental observations}

We show in Fig. 3 the probability distribution functions (PDFs) of $e_{2}$ for several values of $N_{2}$. For each of these curves, one can distinguish two parts: the central peak and the tails. We checked, performing an additional acquisition opening the electrical circuit, that these distributions are not significantly altered and, thus, that the coupling is indeed weak.

We observe that $P\left(e_{2}\right)$ exhibits large wings and a central peak. The first striking observation is that the shape of the wings significantly depends on the number of beads $N_{2}$, although the variance of $e_{2}$ is kept constant. Qualitatively, the occurrence of a central peak can be understood by considering the time series. Focussing on $e_{2}(t)$ for $N_{2}=20$ (Fig. 4, bottom), we observe that for small densities the collisions are independent in time and, moreover, that the blade velocity generally comes back to zero between two successive collisions. This damping of the blade velocity, due to the friction in the motor, naturally leads to the appearance of a central peak in $P\left(e_{2}\right)$. For large $N_{2}$ (=374, Fig. 4, top), the frequency of the collisions is much larger but the effect, even if less important, remains and the central peak is still visible.

In the next section, we propose a tentative model which accounts for both the central peak and the dependence of the wings shape on the bead number, $N_{2}$.

\section{Tentative model}

In order to account, in the simplest model, for the appearance of the central peak and for the change of the shape of the wings, we will first assume that the bead-blade collisions are 


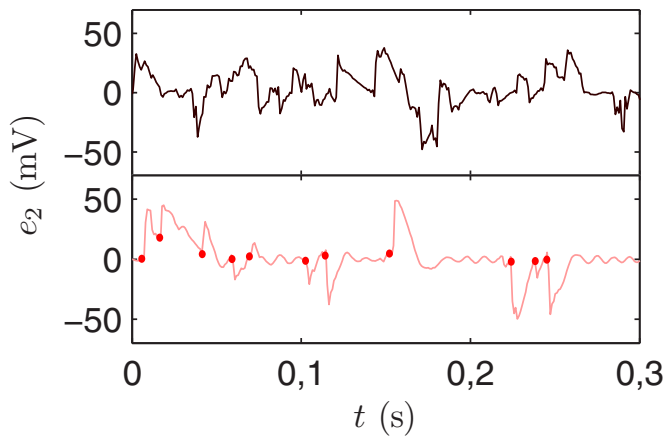

FIG. 4. Examples of temporal signals $e_{2}(t)$ for $N_{2}=374$ (top) and 20 (bottom) beads. Red dots represent collisions of the blade with a bead.

independent, i.e., the blade velocity almost turns back to zero between two successive collisions. Furthermore, we assume that the blade is subjected to fluid friction, such that its velocity after an impact decreases according to $\dot{\theta}(t)=\dot{\theta}_{0} \exp (-t / \tau)$, where $\dot{\theta}_{0}$ is the initial velocity caused by the impact and $\tau$ the characteristic time associated with fluid friction in the dc motor.

Let us now assume that, because of the dynamics of the gas, the successive impacts are separated by the typical time $\tau_{c}$. From the knowledge of the probability distribution $Q\left(\dot{\theta}_{0}\right)$ of the initial velocity $\dot{\theta}_{0}$ right after impact, one can predict the probability distribution of the blade velocity $\dot{\theta}$ by writing $P(\dot{\theta}) d \dot{\theta}=P\left(\dot{\theta}_{0}>\dot{\theta}\right) d t / \tau_{c}$, where $d t=\tau \frac{d \dot{\theta}}{\theta}$ is the duration associated to $d \dot{\theta}$ and integrating over all the possible values of $\dot{\theta}_{0}$ :

$$
P(\dot{\theta})=\frac{\tau}{\tau_{c}|\dot{\theta}|} \frac{\int_{\dot{\theta}}^{\infty} Q\left(\dot{\theta}_{0}\right) d \dot{\theta}_{0}}{\int_{0}^{\infty} Q\left(\dot{\theta}_{0}\right) d \dot{\theta}_{0}} .
$$

The final step is to consider the origin of the distribution $Q\left(\dot{\theta}_{0}\right)$. The latter is directly linked to the distribution $p$ of the velocities of the beads. We assume here $p(v) \propto$ $v^{2} \exp \left[-\left(v / v_{c}\right)^{\mu}\right]$, as proposed in several theoretical studies [21-26]. Consider first the momentum variation due to the impact of a bead on the blade, with normal component $v$ of the velocity, at distance $r$ of the axis:

$$
M \frac{\Delta \dot{\theta}}{\Delta t}=\frac{2 m v}{\Delta t} r .
$$

We assume here for simplicity that the collision is elastic. We thus get that the distribution $Q\left(\dot{\theta}_{0}\right)$ is similar to $p(v)$ with the characteristic angular velocity $\dot{\theta}_{c}=\frac{m R}{M} v_{c}$. As a conclusion, we propose to fit the experimental PDFs by

$$
P(\dot{\theta})=\frac{\tau}{\tau_{c}|\dot{\theta}|} \frac{\int_{\dot{\theta}}^{\infty} \dot{\theta}_{0}^{2} \exp \left[-\left(\dot{\theta}_{0} / \dot{\theta}_{c}\right)^{\mu}\right] d \dot{\theta}_{0}}{\int_{0}^{\infty} \dot{\theta}_{0}^{2} \exp \left[-\left(\dot{\theta}_{0} / \dot{\theta}_{c}\right)^{\mu}\right] d \dot{\theta}_{0}} .
$$

Fitting the experimental data to Eq. (9) with the exponent $\mu$, the characteristic velocity $\dot{\theta}_{c}$, and the prefactor $\tau / \tau_{c}$ [which insures normalization of the $P(\dot{\theta})]$ as free parameters, we observe a good agreement with the experimental distributions (Fig. 5).

The agreement is even excellent for the tails over the whole range of $N_{2}$, with $\mu \simeq 1.45$ (Fig. 6, top). We remember that in the framework of the model, the exponent $\mu$ characterizes the

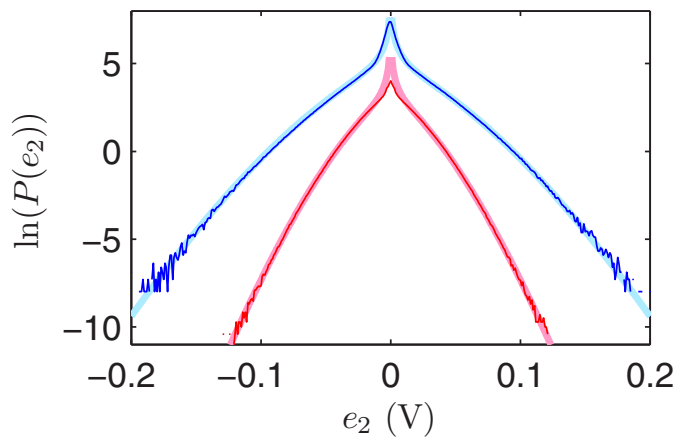

FIG. 5. Probability distribution functions $P\left(e_{2}\right)$ for $N_{2}=20$ (dark blue) and for $N_{2}=374$ beads (dark red). They are fitted by Eq. (9) using $e_{2}=\alpha \dot{\theta}_{2}$ (light blue and light red). Curves are shifted for clarity.

distribution of the bead velocity. We obtain that $\mu$ is constant, independent of the density, in agreement with previous experimental observations in two or three dimensions [27,28] and with theoretical predictions [21-26].

From the interpolation, we also obtain the characteristic angular velocity of the blade $\dot{\theta}_{c}$ which is proportional to the characteristic velocity of the beads. The prefactor depends on experimental parameters only. In the same way $E_{c} \equiv \frac{1}{2} M \dot{\theta}_{c}^{2}=$ $\frac{m R^{2}}{M}\left(\frac{1}{2} m v_{c}^{2}\right)$ is the characteristic kinetic energy of the particles in the gas to a prefactor. Using that $E_{c}$ is proportional to the granular temperature, defined here by $T_{g} \equiv \frac{1}{2} m v_{c}^{2}[29,30]$, we observe, by reporting $E_{c}$ as a function of $N_{2}$ (Fig. 6, bottom), that $T_{g}$ decreases when the density of the gas is increased, even if $E_{k}$, thus the temperature of the blade, is kept constant. The temperature of a blade immersed in the gas is not simply related to the temperature of the latter [27,28,31-33] so that zeroing the flux does not ensure that granular temperatures are equal. Remarking that the energy dissipated by friction in the motor between two collisions should scale like $E_{k} \tau_{c} / \tau$, we can guess that, in a first approximation, $E_{c}=E_{\infty}\left(1+N_{c} / N_{2}\right)$ at constant $E_{k}$. From the experimental data in Fig. 6, bottom, we get $E_{\infty}=3.5 \times 10^{-7} \mathrm{~J}$ and $N_{c} \simeq 40$, the typical number of beads below which dissipation becomes predominant.

Finally, normalizing the distribution $P(\dot{\theta})$, one obtains the ratio $\tau / \tau_{c}$, the typical time of fluid friction divided by the typical time between to successive impacts (Fig. 7). The typical

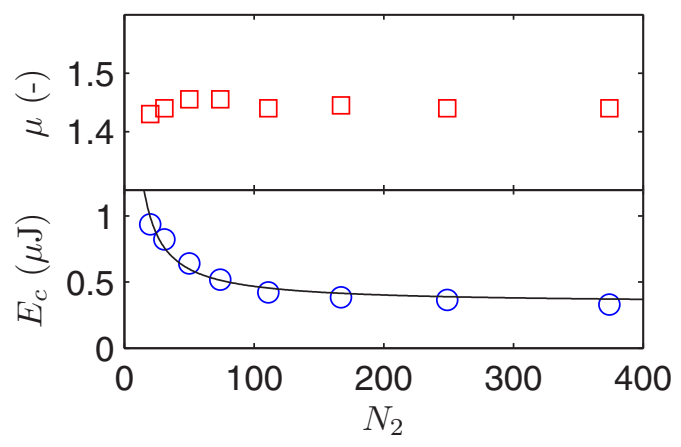

FIG. 6. Parameters $\mu$ (top) and $E_{c}$ (bottom) deduced from the fit of $P\left(e_{2}\right)$ to Eq. (9) as a function of $N_{2}$. Black line: $E_{c}=$ $E_{\infty}\left(1+N_{c} / N_{2}\right)$. 


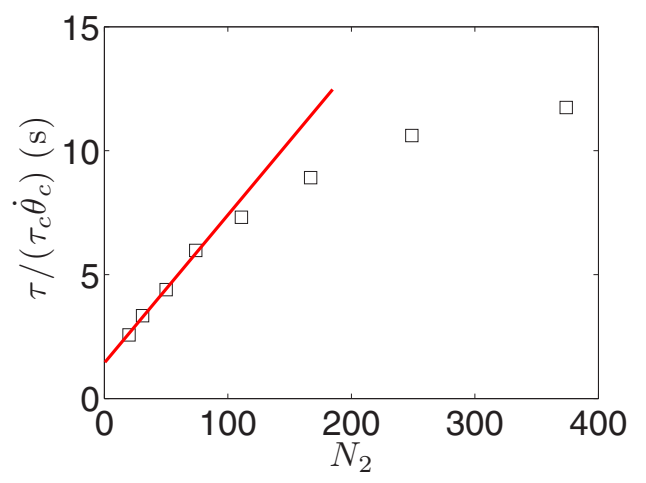

FIG. 7. Ratio $\tau /\left(\tau_{c} \dot{\theta}_{c}\right)$ deduced from Eq. (9) as a function of $N_{2}$.

frequency of the impacts on the blade follows $1 / \tau_{c}=v_{c} \rho S$, where $v_{c}=\dot{\theta}_{c} \frac{M}{R m}, \rho$ is the density of the gas, and $S$ the surface of the blade. Consequently, one would expect $\tau /\left(\tau_{c} \dot{\theta}_{c}\right)$ to be proportional to $N_{2}$ with the coefficient $\frac{\tau S M}{V m R}$, at least in the limit of low densities for which our assumptions are expected to be valid. We observe in Fig. 7 that $\tau /\left(\tau_{c} \dot{\theta}_{c}\right)$ increases with $N_{2}$, the dependence being linear for small densities. The offset is likely due to the stratification of the granular gas in the vessel. In spite of the offset, from the slope and experimental parameters we obtain a crude estimate of the typical time associated with fluid friction, $\tau_{\text {fit }} \simeq 2 \mathrm{~ms}$, which is smaller but compares with the value directly observed in the temporal evolution of $e_{2}$, $\tau_{\text {exp }} \simeq 10 \mathrm{~ms}$ (Fig. 4, bottom).

At this point, we showed that modeling the dissipation, even in a crude way, makes it possible to use the measurements of the blade velocity to assess the properties of the gas. However, we must remark that dissipation is not accounted for in a proper way in various aspects. First, we assumed viscous dissipation in the motor but one can guess that solid friction is also at play, as can be seen in the time series (Fig. 4). Second, focusing on the central peak in Fig. 5, we observe an excellent agreement for small densities only. Obviously the model fails in accounting correctly for the dissipation when the collisions are frequent and occur with the blade still in motion.

As a partial conclusion, we showed that a proper analysis of the velocity fluctuations of the blade, involving the dissipation in the motor, makes it possible to assess properties of the granular gases. In the next section, we consider the exchanges between the two subsystems.

\section{B. Energy flux fluctuations}

From now on, the two motors are connected by a resistor $R$ and the subsystems equilibrated in the sense of zero-mean flux, $\langle\Phi\rangle=0$, as described in Sec. II.

\section{Experimental observations}

We report in Fig. 8 the probability distribution $\mathcal{P}(\Phi)$ of the flux for several values of $N_{2}$ at fixed number of beads $N_{1}$ in the reference system. The first striking observation is that the distribution can be very asymmetric, although the most probable value of the flux $\Phi$ is zero, as well as the mean value $\langle\Phi\rangle=0$. The right wing remains identical when $N_{2}$ is

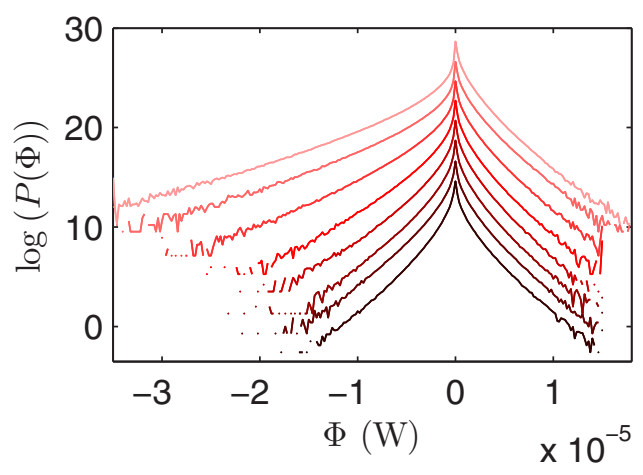

FIG. 8. Probability distribution functions $\mathcal{P}(\Phi)$ for several values of $N_{2}$ (from dark to light red: $N_{2}=374,249,167,111,74,50,30$, and 20 beads). Curves are shifted for clarity.

decreased, but the left wing flattens, leading to a more and more asymmetric and broader distribution.

In order to characterize quantitatively this evolution, we display in Fig. 9 the dependence of the normalized third and forth moments (skewness, $S_{\Phi}$, and kurtosis, $K_{\Phi}$ ) of the PDFs:

$$
S_{\Phi}=\frac{\left\langle\Phi^{3}\right\rangle}{\left\langle\Phi^{2}\right\rangle^{3 / 2}} \quad \text { and } \quad K_{\Phi}=\frac{\left\langle\Phi^{4}\right\rangle}{\left\langle\Phi^{2}\right\rangle^{2}} .
$$

As expected, when the two subsystems are identical $\left(N_{1}=\right.$ $N_{2}=374$ beads), the PDF is symmetric $\left(S_{\Phi}=0\right)$. In addition, we note that $\Phi$ is very intermittent $\left(K_{\Phi} \simeq 20\right.$ to be compared to the values $K_{\Phi}=3$ for a Gaussian distribution and $K_{\Phi}=6$ for an exponential one). We observe a large increase of both the asymmetry and intermittence when $N_{2}$ is decreased.

\section{Tentative model}

A convenient way to regard the structure of the flux is to consider Eq. (6), $\Phi=\left[\left(e_{1}\right)^{2}-\left(e_{2}\right)^{2}\right] / R_{t}$ (with $R_{t}=$ $\left.R+r_{1}+r_{2}\right)$. In the simplest approach, as already proposed in Sec. III A 2, we consider that events in the two subsystems are independent such that $e_{1}$ and $e_{2}$ cannot be nonzero at the same time. In this limit positive values of $\Phi$ are associated to a nonzero value of $e_{1}$, and negative values of $\Phi$ are associated to nonzero value of $e_{2}$. In this picture, the right wing of $\mathcal{P}(\Phi)$ images the reference gas only, whereas the left wing images the gas under study. This is compatible with the observation of an

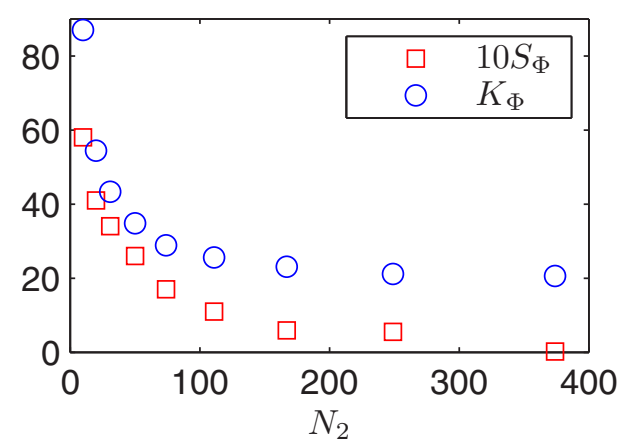

FIG. 9. Skewness ( $S_{\Phi}$, blue circles) and kurtosis $\left(K_{\Phi}\right.$, red squares) of $P(\Phi)$ as a function of $N_{2}$. 


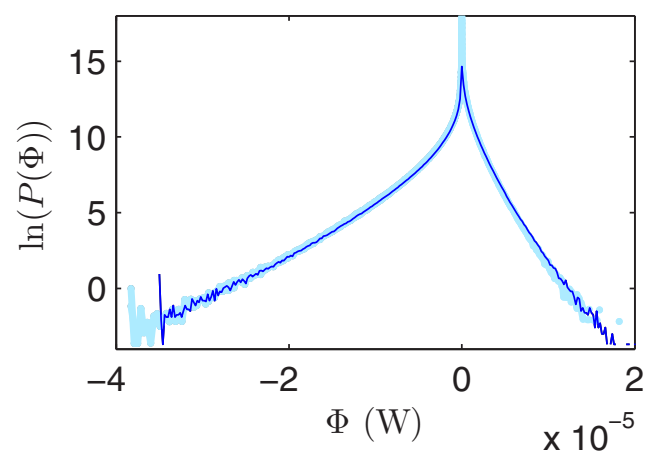

FIG. 10. Distribution $\mathcal{P}(\Phi)$ (dark blue) compared to its estimate from Eqs. (10) (light blue) $\left[N_{1}=374-N_{2}=20\right]$.

unaltered right wing of the distribution when $N_{2}$ is decreased (Fig. 9). This is also compatible with the flattening of the left wing when $\mathrm{N}_{2}$ is decreased.

If the picture holds correct, one would recover the distribution $\mathcal{P}(\Phi)$ from the distribution $P\left(\dot{\theta}_{1}\right)$ and $P\left(\dot{\theta}_{2}\right)$ using a simple change of variable based on Eq. (6):

$$
\begin{aligned}
& \mathcal{P}(\Phi)=\frac{R_{t}}{2 \dot{\theta}_{1}} P\left(\dot{\theta}_{1}\right) \quad \text { for } \quad \Phi>0, \\
& \mathcal{P}(\Phi)=\frac{R_{t}}{2 \dot{\theta}_{2}} P\left(\dot{\theta}_{2}\right) \quad \text { for } \quad \Phi<0 .
\end{aligned}
$$

We observe in Fig. 10 an excellent agreement between the experimental distribution of $\Phi$ and its estimate from the experimental distribution of $\dot{\theta}_{1,2}$ using Eq. (10). The agreement is excellent for all numbers $N_{2}$ explored experimentally.

As a conclusion the knowledge of the velocity fluctuations of the two blades allows, in spite of a strong assumption, recovery of the main feature of the flux between the two subsystems.

\section{DISCUSSION AND CONCLUSION}

We reported on an experimental realization of two coupled nonequilibrium steady-state systems. In practice, two granular gases are coupled by electromechanical devices: a blade attached to the rotor of a dc motor is immersed in each of the gases. The motors are connected one to another through a resistor.
We measured the probability distribution of the blade velocity. The shape of the latter depends on the density of the gas. However, proper account of the experimental device, especially of the friction in the motors, allows us to access the properties of the gas itself. In particular, assuming the distribution of the velocity $v$ of the particles in the gas to be of the form $p(v) \propto v^{2} \exp \left[-\left(v / v_{c}\right)^{\mu}\right]$, we obtain the exponent $\mu \simeq 1.45$, independent of the density of the gas (Sec. III A 2).

In a second step, we measured the energy flux between the two subsystems and showed that the structure of the fluctuations can be recovered using a simple change of variable (Sec. III B 2). We note the qualitative similarity between the probability distributions presented here and those obtained for the power injected in a certain number of NESS (plate wave turbulence [34]; capillary wave turbulence [10]; see also [17,35]). However, we point out that all these studies concern the power injected to compensate the dissipation, i.e., sustain the NESS, whereas we considered the exchanges between two NESS. This resemblance might not be fortuitous.

The main physical conclusion is that the dynamics of the "thermometer," the blade, becomes more and more intermittent when the density of the gas is reduced, whereas the structure of the fluctuations of the particle velocities remains unchanged. When the two gases are coupled, even if zero average flux between them is imposed $(\langle\Phi\rangle=0)$, one observes that the typical energy of the particles in the gas having smaller density is larger (Sec. III A 2). This can be understood qualitatively by an increase of the relative effect of the dissipation in the motors. This increase explains, by itself, the increase of the intermittent character of the blade dynamics.

We must, however, comment that we used in our analysis crude approximations. First of all, in order to obtain the velocity statistics in the gas from the velocity fluctuations of the blade, we assumed that the blade velocity comes back to zero between two successive collisions. This is, however, not true for the largest densities considered here. Moreover, we considered the case of viscous damping only and forgot any potential effect of solid friction. In spite of these crude assumptions, our analysis works surprisingly well. Second, considering the flux between the two subsystems, we again assumed that effects of collisions in the two gases do not overlap. This is certainly not true, but the arguments are enough to explain the shape of the flux distribution. There is clearly a need for a further theoretical effort to take into account all these effects.
[1] S.-I. Sasa and H. Tasaki, J. Stat. Phys. 125, 125 (2006).

[2] S. Aumaître, S. Fauve, S. McNamara, and P. Poggi, Eur. Phys. J. B 19, 449 (2001).

[3] R. Labbe, J. F. Pinton, and S. Fauve, J. Phys. II 6, 1099 (1996).

[4] M. M. Bandi, S. G. Chumakov, and C. Connaughton, Phys. Rev. E 79, 016309 (2009).

[5] G. Ahlers, S. Grossmann, and D. Lohse, Rev. Mod. Phys. 81, 503 (2009).

[6] N. Garnier and S. Ciliberto, Phys. Rev. E 71, 060101(R) (2005).
[7] C. Falcón and E. Falcon, Phys. Rev. E 79, 041110 (2009).

[8] M. Bonaldi, L. Conti, P. De Gregorio, L. Rondoni, G. Vedovato, A. Vinante, M. Bignotto, M. Cerdonio, P. Falferi, N. Liguori, S. Longo, R. Mezzena, A. Ortolan, G. A. Prodi, F. Salemi, L. Taffarello, S. Vitale, and J. P. Zendri, Phys. Rev. Lett. 103, 010601 (2009)

[9] O. Cadot, C. Touzé, and A. Boudaoud, Phys. Rev. E 82, 046211 (2010).

[10] E. Falcon, S. Aumaître, C. Falcón, C. Laroche, and S. Fauve, Phys. Rev. Lett. 100, 064503 (2008).

[11] U. Seifert, Rep. Prog. Phys. 75, 126001 (2012). 
[12] J. Prost, J.-F. Joanny, and J. M. R. Parrondo, Phys. Rev. Lett. 103, 090601 (2009).

[13] M. C. Marchetti, J. F. Joanny, S. Ramaswamy, T. B. Liverpool, J. Prost, M. Rao, and R. A. Simha, Rev. Mod. Phys. 85, 1143 (2013).

[14] É. Fodor, K. Kanazawa, H. Hayakawa, P. Visco, and F. van Wijland, Phys. Rev. E 90, 042724 (2014).

[15] A. Mehta, Granular Physics (Cambridge University Press, Cambridge, UK, 2007).

[16] I. S. Aranson and J. S. Olafsen, Phys. Rev. E 66, 061302 (2002).

[17] S. Aumaître, J. Farago, S. Fauve, and S. Mc Namara, Eur. Phys. J. B 42, 255 (2004).

[18] P. Visco, A. Puglisi, A. Barrat, E. Trizac, and F. van Wijland, Europhys. Lett. 72, 55 (2005).

[19] S. Aumaître, A. Alastuey, and S. Fauve, Eur. Phys. J. B 54, 263 (2006).

[20] C.-É. Lecomte and A. Naert, J. Stat. Mech.: Theory Exp. (2014) P11004.

[21] T. P. C. van Noije and M. H. Ernst, Gran. Matt. 1, 57 (1998).

[22] A. Barrat, T. Biben, Z. Racz, E. Trizac, and F. van Wijland, J. Phys. A: Math. Gen. 35, 463 (2002).
[23] A. Barrat and E. Trizac, Eur. Phys. J. E: Soft Matter Biol. Phys. 11, 99 (2003)

[24] A. Barrat, E. Trizac, and M. H. Ernst, J. Phys.: Condens. Matter 17, S2429 (2005).

[25] M. H. Ernst and R. Brito, J. Stat. Phys. 109, 407 (2002).

[26] M. H. Ernst and R. Brito, Phys. Rev. E 65, 040301(R) (2002).

[27] A. Gnoli, A. Puglisi, and H. Touchette, Europhys. Lett. 102, 14002 (2013).

[28] T. G. Sano and H. Hayakawa, Phys. Rev. E 89, 032104 (2014).

[29] A. Baldassarri, A. Barrat, G. D’Anna, V. Loreto, P. Mayor, and A. Puglisi, J. Phys.: Condens. Matter 17, S2405 (2005).

[30] I. Goldhirsch, Powder Technol. 182, 130 (2008).

[31] A. Gnoli, A. Puglisi, A. Sarracino, and A. Vulpiani, PLoS One 9, e93720 (2014).

[32] J. Talbot, R. D. Wildman, and P. Viot, Phys. Rev. Lett. 107, 138001 (2011).

[33] T. G. Sano, K. Kanazawa, and H. Hayakawa, Phys. Rev. E 94, 032910 (2016).

[34] O. Cadot, A. Boudaoud, and C. Touzé, Eur. Phys. J. B 66 (2008).

[35] M. M. Bandi and C. Connaughton, Phys. Rev. E 77, 036318 (2008). 